\title{
Detection of Streptococcus Pneumoniae and elimination of carryover contamination by multiple cross displacement amplification coupled with antarctic thermal sensitive uracil-DNA-glycosylase
}

\section{Linlin Yan}

Peking University Shougang Hospital

\section{Fan Zhao}

Peking University Shougang Hospital

Lina Niu

Hainan Medical University

\section{Yu Cai}

Peking University Shougang Hospital

Lei Wu

Peking University Shougang Hospital

Xiaoxue Zhu

Peking University Shougang Hospital

Yuanyuan Wu

Peking University Shougang Hospital

Jinqing Nong

Peking University Shougang Hospital

Shoukui Hu ( $\nabla$ shoukuihu@163.com )

\section{Research article}

Keywords: Streptococcus pneumoniae; Multiple cross displacement amplification; Lateral flow biosensor; Antarctic thermal sensitive uracil-DNA glycosylase (AUDG); False-positive result

Posted Date: April 19th, 2019

DOI: https://doi.org/10.21203/rs.2.9259/v1

License: (c) (1) This work is licensed under a Creative Commons Attribution 4.0 International License. Read Full License 


\section{Abstract}

Background Streptococcus pneumoniae (S. pneumoniae), as an important human pathogenic bacterium, causes high mortality and morbidity, especially among children younger than 5 years. Accurate and rapid detection of S. pneumoniae is critical important. Methods A ply gene-based multiple cross displacement amplification (MCDA), which amplifies DNA under isothermal conditions $\left(65^{\circ} \mathrm{C}\right)$ for 40 min, was established for accurate and rapid detection of S. pneumoniae. The amplicons were indicated using lateral flow biosensor (LFB). Results The MCDA assay could detect as little as $10 \mathrm{fg}$ purified S. pneumoniae genomic DNA, and $447 \mathrm{CFU} / \mathrm{mL}$ spiked sputum samples with a sensitivity 100 times that of PCR. The specificity of MCDA primer targeting ply gene was validated using $15 \mathrm{~S}$. pneumoniae strains and 25 non-S. pneumoniae strains, indicating that ply gene-based MCDA assay was highly selective for S. pneumoniae. Moreover, the MCDA assay coupled with antarctic thermal sensitive uracil-DNAglycosylase (AUDG) can effectively eliminate the carryover contamination, and then preventing falsepositive results. Conclusion MCDA assay in combination with AUDG was a simple, rapid, sensitive, specific and reliable method for the diagnosis of S. pneumoniae infection, which appears to be a valuable tool for clinical screening.

\section{Background}

Streptococcus pneumoniae (S. pneumoniae) is a gram-positive bacterium, regularly colonize in the upper respiratory tract, which is the most common pathogen of human meningitis, septicemia, communityacquired pneumonia, otitis media and sinusitis [1, 2]. According to the 2005 data of World Health Organization (WHO), S. pneumoniae infection causes 1.6 million deaths worldwide each year, of which 700,000 to 1 million are children under the age of 5 , and mostly from developing countries [3]. Thus, accurate and rapid detection of $S$. pneumoniae is the primary task of preventing spread of the pathogen and reducing the harm caused by disease.

The traditional detection technique for $S$. pneumoniae is bacterial culture-colonial morphology biochemical-biochemical examination, which is time-consuming, usually requiring three or more days. It is of vital importance for clinical treatment to accurately and rapidly detecting $S$. pneumoniae. Molecular biology techniques, such as polymerase chain reaction (PCR)-based assays have been used for detection of $S$. pneumoniae with primers specific to pneumolysin [4-6]. Pneumolysin, which is encoded by the ply gene, is a virulence factor from S. pneumoniae and is involved in pathogenesis [7, 8], representing the potential diagnostic target. However, the PCR reaction system is complex, complicated to perform, and requiring expensive thermal cycle apparatus and professional technicians [9]. Thus, PCRbased assays are not available in some backward clinical laboratories and "on-site" detection.

In recent years, Wang et al. [10] devised a novel nucleic acid isothermal amplification method, multiple cross displacement amplification (MCDA). Compared with PCR, MCDA has superior performance, which amplifies DNA under isothermal temperature $\left(60^{\circ} \mathrm{C}-69^{\circ} \mathrm{C}\right)$ with higher efficiency, specificity and only a simple thermostatic equipment is required. This method has been used for detecting Klebsiella 
pneumoniae [11], Vibrio parahaemolyticus [12], Listeria monocytogenes [13] and so on. MCDA reaction system requires ten specially designed primers that recognize ten distinct regions of target gene and the reaction can be completed within $40 \mathrm{~min}$. Therefore, MCDA is a potential valuable method for rapid detection of S. pneumoniae, especially when resource settings are limited. In addition, four detection methods for MCDA products including colorimetric indicator, real-time turbidity, lateral flow biosensor (LFB) and gel electrophoresis have been performed [14].

In this study, we established a highly sensitive and specific MCDA assay for accurate and rapid detection of $S$. pneumoniae. Ply gene, a $S$. pneumoniaespecific gene that encodes pneumolysin, is used as the target gene. We determined the optimal temperature, sensitivity, specificity and the clinical sensitivity of $S$. pneumoniae MCDA assay. Furthermore, MCDA was coupled with antarctic thermal sensitive uracil-DNA glycosylase (AUDG) to eliminate carryover contamination and prevent the false-positive results.

\section{Methods}

\section{Primer design}

Pneumolysin, a virulence factor, encoded by the ply gene, is a specific target for the detection of $S$. pneumoniae. Five pairs of MCDA primers were designed on the ply gene from the S. pneumoniae standard strain of ATCC49619 according the principle of MCDA developed by Wang et al $[13,14]$. Primer Primer 5.0 and PrimerExploer V4 were used. The pneumolysin $(p / y)$-encoding primer sets are listed in Table 3 and Figure 5. The 5'- ends of the primers C1 and D1 were labeled with fluorescein isothiocyanate (FITC) and biotin, respectively. Primers were synthesized in Aoke Dingsheng Biotechnology Co., Ltd. (Beijing, China).

\section{Bacteria strains}

The bacteria strains used in this study included 15 S. pneumoniae strains and 25 non-S. pneumoniae strains (Table 1). The QIAamp DNA minikits (Hilden, Germany) was used for the extraction of genomic DNA from bacteria strains. Protocols were carried out in accordance with the manufacturer's instructions. The S. pneumoniae standard strain ATCC49619 was used for confirmation of the performance, determination of the optimal temperature and analysis of sensitivity. DNA of S. pneumoniae ATCC49619 was serially diluted $(10 \mathrm{ng}, 10 \mathrm{pg}, 1 \mathrm{pg}, 100 \mathrm{fg}, 10 \mathrm{fg}, 1 \mathrm{fg}$ and $0.1 \mathrm{fg} / \mu \mathrm{L})$ for sensitivity analysis.

\section{S. pneumoniae MCDA assay}

The S. pneumoniae MCDA assay was conducted based on previously published methods of Wang et al. $[13,14]$. The isothermal amplification kits (Haitaizhengyuan, Beijing, China) was used in this study. $S$. pneumoniae MCDA assay was carried out in a $25 \mu \mathrm{l}$ reaction mixture including $1 \mu$ l of DNA template, $12.5 \mu$ l of $2 \times$ Reaction buffer, $0.4 \mu \mathrm{M}$ of $\mathrm{F} 1$ and $\mathrm{F} 2,1.6 \mu \mathrm{M}$ of $\mathrm{CP} 1$ and $\mathrm{CP} 2,0.8 \mu \mathrm{M}$ of $\mathrm{C} 1\left(\mathrm{C} 1^{*}\right), \mathrm{C} 2, \mathrm{D} 1$ (D1*), D2, R1 and R2, and $1 \mu$ l of Bst DNA polymerase (10U). DNA of Staphylococcus aureus and Salmonella typhii were used as negative controls. Distilled water was used as blank control. The reaction systems were isothermally amplified at $63^{\circ} \mathrm{C}$ for $40 \mathrm{~min}$, followed by $85^{\circ} \mathrm{C}$ for 5 min to stop the amplification. 
In this study, four methods were used to determine the amplification results, including colorimetric indicator (Malachite Green, MG), turbidimeter (Loopamp Realtime Turbidimeter LA-320C), lateral flow biosensor (LFB) and $2 \%$ agarose gel electrophoresis. The above four methods were performed as previously described [14].

\section{Optimal temperature of $S$. pneumoniae MCDA assay}

The optimal amplification temperature of $S$. pneumoniae MCDA assay was determined at isothermal temperature ranging from 62 to $67^{\circ} \mathrm{C}$ with $1{ }^{\circ} \mathrm{C}$ intervals. DNA templates of Staphylococcus aureus and Salmonella typhi were used as negative control, and distilled water was used as blank control. The temperature that contributed to produce higher amplification products and occur turbidity earlier was considered for the optimal temperature. The experiment was repeated three times.

\section{Sensitivity of S. pneumoniae MCDA assay}

Sensitivity of MCDA assay was determined using serial dilutions of S. pneumoniae ATCC49619 genomic DNA. A total of 7 different concentrations of DNA templates (10ng, 10pg, 1pg, 100fg, 10fg, $1 \mathrm{fg}, 0.1 \mathrm{fg} / \mu \mathrm{L}$ ) were acquired. $1 \mu \mathrm{l}$ of each dilution were added into the amplication mixture and then to be amplified at optimal temperature for 40min. Color indicator, real time turbidity, LFB detection and gel electrophoresis were conducted to analyze the MCDA products and then determine the limit of detection of $S$. pneumoniae MCDA assay. Distilled water was used as blank control. The sensitivity experiment was repeated in triplicate.

\section{Eliminating carryover contamination by using AUDG enzyme}

The $S$. pneumoniae MCDA products in the absence of AUDG enzyme were quantified using ultraviolet spectrophotometer (NanoDrop ND-1000, Calibre, China), and then to be serially diluted from $1 \times 10-10$ to $1 \times 10-20 \mathrm{~g} / \mu \mathrm{L}$. These dilutions were served as the source of simulated carryover contamination and were used as templates in S. pneumoniae MCDA assay. To demonstrate that simulated carryover contamination (UTP-incorporated products) can contaminate the MCDA reaction and to confirm that AUDG enzyme can eliminate the false-positive results caused by carryover contaminants, the sensitivity of MCDA-AUDG and MCDA were compared by using the above serial diluted MCDA products $(1 \mu \mathrm{l})$.

\section{Specificity of S. pneumoniae MCDA assay}

To determine the specificity of $S$. pneumoniae MCDA assay, DNA templates from 40 bacteria strains including 15 S. pneumoniae strains and 25 non-S. pneumoniae strains were applied to the MCDA assay (Table 1). The products were analyzed using color indicator and LFB detection. The specificity experiment was repeated three times.

\section{Evaluation of clinical sensitivity of $S$. pneumoniae MCDA assay}


The sensitivity of S. pneumoniae MCDA assay in clinical application was evaluated by artificially adding serial dilutions of colony forming units (CFUs) of S. pneumoniae ATCC49619 into the sputum samples. The single colony of ATCC49619 was enriched and cultured. Then, the number of ATCC49619 CFUs was counted, and cultured suspensions were added into the $S$. pneumoniae negative sputum samples. The concentration of ATCC49619 in sputum samples was adjusted to $4.47 \times 100,4.47 \times 101,4.47 \times 102$, $4.47 \times 103,4.47 \times 104,4.47 \times 105,4.47 \times 106 \mathrm{CFU} / \mathrm{mL}$. Then, $100 \mu \mathrm{L}$ of artificially contaminated sputum samples were subjected to extract the genomic DNA, which were eluted in $10 \mu \mathrm{L}$ of Qiagen elution buffer (Qiagen, Germany). Next, $1 \mu \mathrm{L}$ of DNA templates were used for MCDA assay and the PCR method was also adopted. The experiment was performed three times independently.

\section{Results}

\section{Confirmation of S. pneumoniae MCDA products.}

To confirm the feasibility of the MCDA primers in detecting S. pneumoniae, MCDA amplification was performed at $63^{\circ} \mathrm{C}$ for 40 min with and without target DNA. The MCDA product of $S$. pneumoniae ATCC49619 was detected visually as lake green color in the tube (Fig.1A), two red lines (control line and test line) on the LFB (Fig.1B) and ladder-like bands on the gel (Fig.1C), which indicated the amplification with target sequence. While the MCDA products of negative control and blank control were present as colorless (Fig.1A), only one red line (control line) (Fig.1B) and no ladder-like bands (Fig.1C). Our results indicated that the $S$. pneumoniae-specific primers targeting ply gene were suitable for MCDA assay.

\section{Optimal temperature of $S$. pneumoniae MCDA assay.}

To identify the optimal amplification temperature for S. pneumoniae MCDA assay, S. pneumoniae ATCC49619 DNA was used ( $1 \mathrm{pg} /$ reaction) to be amplified at different temperatures ranging from $62^{\circ} \mathrm{C}$ to $67^{\circ} \mathrm{C}$ at $1^{\circ} \mathrm{C}$ intervals. The real time turbidity of the reactions was monitored. From the Kinetic curves as shown in Figure 2, we found that $65^{\circ} \mathrm{C}$ was the optimal amplification temperature, because the turbidity of the reaction occurred earlier and the amount of MCDA products were higher than other temperatures (Fig. 2D). $65^{\circ} \mathrm{C}$ was used in the subsequent studies.

\section{Sensitivity of $S$. pneumoniae MCDA assay.}

To determine the limit of detection (LoD), serial dilutions of S. pneumoniae ATCC49619 DNA (10ng, 10pg, $1 \mathrm{pg}, 100 \mathrm{fg}, 10 \mathrm{fg}, 1 \mathrm{fg}, 0.1 \mathrm{fg} / \mathrm{\mu L}$ ) were subjected to be amplified at $65^{\circ} \mathrm{C}$. The results showed that the LoD of $S$. pneumoniae MCDA assay was $10 \mathrm{fg}$ (Fig.3). The positive amplicons were represented in the color indicator (Fig.3A), real-time turbidity (Fig.3B), LFB (Fig.3C) and gel electrophoresis (Fig.3D) as lake green, turbidity curve, two red lines and ladder-like bands, respectively. It is worth noting that the results of the above four methods were completely consistent.

\section{MCDA assay combined with AUDG enzyme eliminates false-positive results caused by carryover contamination.}


To determine whether carryover contamination from UTP-incorporated MCDA products would contaminate the MCDA reactions, we evaluated the sensitivity of MCDA-AUDG and MCDA using serial diluted MCDA products with the concentrations ranging from $1 \times 10-10$ to $1 \times 10-20 \mathrm{~g} / \mu \mathrm{L}$. Our results showed that MCDA-AUDG can only detect $1 \times 10-14 \mathrm{~g} / \mu \mathrm{L}$ or more of simulated carryover contamination (Fig.4). Without AUDG enzyme, MCDA can detect as little as $1 \times 10-19 \mathrm{~g} / \mu \mathrm{L}$ of simulated carryover contamination (Fig.4). These results indicated that carryover contamination with low concentrations $(1 \times 10-19 \mathrm{~g} / \mu \mathrm{L})$ can cause false-positive results. In addition, our results also demonstrated that AUDG enzyme is able to eliminate the carryover contamination to a certain extent. Therefore, MCDA assay coupled with AUDG enzyme can significantly reduce the possibility of false-positive amplifications of $S$. pneumoniae.

\section{Specificity of $S$. pneumoniae MCDA assay.}

To determine the specificity of $S$. pneumoniae MCDA assay with AUDG digestion in detecting $S$. pneumoniae, DNA from 15 S. pneumoniae strains and 25 non-S. pneumoniae strains were tested. All of the DNA from S. pneumoniae strains showed positive results, while the DNA from other Streptococcus species and non-Streptococcus strains showed negative results (Table 1). These results indicated that the MCDA assay with AUDG enzyme was $100 \%$ specific for S. pneumoniae detection.

\section{Clinical sensitivity of $S$. pneumoniae MCDA assay in sputum samples.}

To explore the sensitivity of MCDA assay with AUDG digestion in clinical application, the artificially contaminated sputum samples with different concentrations of S. pneumoniae ATCC49619 were analyzed by using MCDA-AUDG assay. The MCDA-AUDG assay occurred positive amplification when the amount of $S$. pneumoniae in the spiked sputum samples were more than $447 \mathrm{CFU} / \mathrm{ml}$ (equivalent to 2.9 CFU per reaction) (Table 2). The sensitivity of MCDA-AUDG assay was 100 times that of PCR, the LoD of PCR was $44700 \mathrm{CFU} / \mathrm{ml}$ (equivalent to $290 \mathrm{CFU}$ per reaction) (Table 2).

\section{Discussion}

S. pneumoniae, an important human pathogenic bacterium, causes high mortality and morbidity. Statistics show that in China, the cases of pneumococcal infection account for $12 \%$ of the global cases, which is also one of the countries with the highest deaths caused by pneumococcal infection in children younger than 5 years [15]. Because of the severity of $S$. pneumoniae infection, simple and accurate methods are needed to detect $S$. pneumoniae and timely guide clinical treatment. Mitsuko et al. has reported a rapid method based on loop-mediated isothermal amplification (LAMP) for the diagnosis of $S$. pneumoniae infection, which is highly selective because it uses four primers to recognize six different regions of the target gene $[16,17]$. Studies have reported that increasing the primers of isothermal amplification could improve the sensitivity [18]. This study developed a novel method, MCDA in combination with AUDG, to specifically detect $S$. pneumoniae and prevent false positive results.

Within the present study, we designed five pairs primers targeting ten distinct regions of the ply gene. Ply gene, which encodes pneumolysin, a well-characterized virulence factor of $S$. pneumoniae and 
participating in the pathogenesis, has been used as the specific target gene of S. pneumoniae $[7,8]$. Our results demonstrated that the primers are highly specific for the detection of $S$. pneumoniae. Only the DNA from S. pneumoniae strains were positively amplified, while the DNA from other Streptococcus species and non-Streptococcus strains were not amplified (Table 1). Therefore, the selectivity of the MCDA assay is extremely high in detecting S. pneumoniae.

In addition, we evaluated the sensitivity of MCDA assay by testing serial dilutions of $S$. pneumoniae ATCC49619 DNA. The detection limit of MCDA assay was determined to be $10 \mathrm{fg}$ (Fig.3). It has been reported that LAMP can detect $S$. pneumoniae as few as $25 \mathrm{fg}$ [19] or $20 \mathrm{fg}$ [20]. We also assessed the clinical sensitivity of this assay by using spiked sputum samples. The MCDA can detect $S$. pneumoniae as low as $447 \mathrm{CFU} / \mathrm{ml}$ (equivalent to $2.9 \mathrm{CFU}$ per reaction), which is similar with that of LAMP [19]. Thus, MCDA appears to be a sensitive method of detecting $S$. pneumoniae infection.

However, owing to the high sensitivity of MCDA assay, false-positive results caused by carryover contamination became a barrier to accurate and rapid diagnosis of $S$. pneumoniae. Moreover, because of the opening indicating operation of MCDA products (such as LFB), the aerosol droplets containing high concentration of products cannot be avoided. In this study, our results showed that trace amounts of carryover contamination $(1 \times 10-19 \mathrm{~g} / \mu \mathrm{L})$ can lead to false-positive amplification. Thus, we prevent the false-positive results arising from carryover contamination by performing AUDG enzyme digestion, which specifically cleave uracil bases and permit the nature templates to be amplified normally [11, 19], and can eliminate as much as $1 \times 10-15 \mathrm{~g} / \mu \mathrm{L}$ of simulated contaminants. These results suggested that MCDAAUDG assay can effectively eliminate carryover contamination and then reduce false-positive amplications.

About products analysis, four methods including colorimetric indicator, real-time turbidity, LFB and gel electrophoresis were used. The results of these four methods were highly consistent. However, comparing with conventional analysis methods, LFB was probably the optimal monitoring method. In the MCDA system, one primer was labeled with FITC and one primer was labeled with biotin. Subsequently, the positive amplification products were labeled with FITC and biotin. Within the LFB, the FITC and biotin labeled products combined the anti-FITC antibody immobilized on the test line and dye streptavidin coated on the control line, respectively, and the results are displayed as two red lines within 2 minutes. The advantages of LFB included simple to use, rapid, cost-effective, objective and no equipment requirement. Thus, LFB is an effective method for the products analysis of MCDA assay.

In conclusion, we established a simple, rapid, sensitive, specific and reliable MCDA-LFB method in combination with AUDG for the diagnosis of $S$. pneumoniae and prevention of false-positive results caused by carryover contamination. LFB detection provided a potential tool for accurate and rapid detection of $S$. pneumoniae. The sensitivity, specificity and clinical sensitivity of MCDA assay in the diagnosis of $S$. pneumoniae were evaluated by using pure cultures and spiked sputum samples. Therefore, the MCDA-LFB coupled with AUDG is potentially a valuable tool for the detection of $S$. pneumoniae. 


\section{Abbreviations}

AUDG: Antarctic thermal sensitive uracil-DNA glycosylase

CFUs: colony forming units

FITC: fluorescein isothiocyanate

LAMP: loop-mediated isothermal amplification

LFB: lateral flow biosensor

LoD: limit of detection

MCDA: multiple cross displacement amplification

MG: Malachite Green

PCR: polymerase chain reaction

Ply: pneumolysin

WHO: World Health Organization

\section{Declarations}

\section{Acknowledgments}

Not applicable

\section{Funding}

This work was supported by the Beijing Natural Science Foundation [grant number 7192240], the National Natural Science Foundation of China [grant number 81760376] and the Peking University Shougang Hospital [grant numbers 2017-Yuan-LC-08, SGYYZ201701].

\section{Availability of data and materials}

The datasets used and/or analysed during the current study are available from the corresponding author on reasonable request.

\section{Authors' contributions}

$L Y, F Z, L N, S H$ designed the research and wrote the manuscript; LY, FZ and LN performed the experiments and analyzed the data; $\mathrm{YC}, \mathrm{LW}, \mathrm{XZ}, \mathrm{YW}$ and $\mathrm{JN}$ contributed the reagents and materials; $\mathrm{SH}$ supervised the study. All authors reviewed the manuscript. 


\section{Ethics approval and consent to participate}

This study was carried out in accordance with the recommendations of Declaration of Helsinki, Ethics Committee of Peking University Shougang Hospital with written informed consent from all subjects. All subjects gave written informed consent in accordance with the Declaration of Helsinki. The protocol was approved by the Ethics Committee of Peking University Shougang Hospital.

\section{Consent for publication}

Not applicable

\section{Competing interests}

The authors declare that they have no competing interests.

\section{References}

1. Brown PD, Lerner SA. Community-acquired pneumonia. Lancet. 1998; 352:1295-302.

2. Yao KH, Yang YH. Streptococcus pneumoniae diseases in Chinese children: past, present and future. Vaccine. 2008; 26:4425-33.

3. Organization WH. Pneumococcal conjugate vaccine for childhood immunization-WHO position paper. Wkly Epidemiol Rec. 2007; 82:93-104.

4. Rudolph KM, Parkinson AJ, Black CM, Mayer LW. Evaluation of polymerase chain reaction for diagnosis of pneumococcal pneumonia. Journal of clinical microbiology. 1993; 31:2661-6.

5. Salo P, Ortqvist A, Leinonen M. Diagnosis of bacteremic pneumococcal pneumonia by amplification of pneumolysin gene fragment in serum. The Journal of infectious diseases. 1995; 171:479-82.

6. Wheeler J, Freeman R, Steward M, Henderson K, Lee MJ, Piggott NH, et al. Detection of pneumolysin in sputum. Journal of medical microbiology. 1999; 48:863-6.

7. Rossjohn J, Gilbert RJ, Crane D, Morgan PJ, Mitchell TJ, Rowe AJ, et al. The molecular mechanism of pneumolysin, a virulence factor from Streptococcus pneumoniae. Journal of molecular biology. 1998; 284:449-61.

8. Berry AM, Lock RA, Hansman D, Paton JC. Contribution of autolysin to virulence of Streptococcus pneumoniae. Infection and immunity. 1989; 57:2324-30.

9. Gillespie SH. The role of the molecular laboratory in the investigation of Streptococcus pneumoniae infections. Seminars in respiratory infections. 1999; 14:269-75.

10. Wang Y, Wang Y, Ma AJ, Li DX, Luo LJ, Liu DX, et al. Rapid and Sensitive Isothermal Detection of Nucleic-acid Sequence by Multiple Cross Displacement Amplification. Scientific reports. 2015; 5:11902. 
11. Wang Y, Yan W, Wang Y, Xu J, Ye C. Rapid, sensitive and reliable detection of Klebsiella pneumoniae by label-free multiple cross displacement amplification coupled with nanoparticles-based biosensor. Journal of microbiological methods. 2018; 149:80-88.

12. Wang Y, Li H, Li D, Li K, Wang Y, Xu J, et al. Multiple Cross Displacement Amplification Combined with Gold Nanoparticle-Based Lateral Flow Biosensor for Detection of Vibrio parahaemolyticus. Frontiers in microbiology. 2016; 7:2047.

13. Wang Y, Li H, Wang Y, Li H, Luo L, Xu J, et al. Development of multiple cross displacement amplification label-based gold nanoparticles lateral flow biosensor for detection of Listeria monocytogenes. International journal of nanomedicine. 2017; 12:473-86.

14. Wang $\mathrm{Y}$, Wang $\mathrm{Y}$, Wang $\mathrm{H}, \mathrm{Xu} \mathrm{J}, \mathrm{Ye} \mathrm{C}$. A label-free technique for accurate detection of nucleic acidbased self-avoiding molecular recognition systems supplemented multiple cross-displacement amplification and nanoparticles based biosensor. Artificial cells, nanomedicine, and biotechnology. 2018; 46:1671-84.

15. O'Brien KL, Wolfson LJ, Watt JP, Henkle E, Deloria-Knoll M, McCall N, et al. Burden of disease caused by Streptococcus pneumoniae in children younger than 5 years: global estimates. Lancet. 2009 ; 374:893902.

16. Seki M, Yamashita Y, Torigoe H, Tsuda H, Sato S, Maeno M. Loop-mediated isothermal amplification method targeting the lytA gene for detection of Streptococcus pneumoniae. Journal of clinical microbiology. 2005; 43:1581-6.

17. Notomi T, Okayama H, Masubuchi H, Yonekawa T, Watanabe K, Amino N, et al. Loop-mediated isothermal amplification of DNA. Nucleic acids research. 2000; 28:E63.

18. Soo PC, Tseng CC, Ling SR, Liou ML, Liu CC, Chao HJ, et al. Rapid and sensitive detection of Acinetobacter baumannii using loop-mediated isothermal amplification. Journal of microbiological methods. 2013; 92:197-200.

19. Wang Y, Wang Y, Li D, Xu J, Ye C. Detection of nucleic acids and elimination of carryover contamination by using loop-mediated isothermal amplification and antarctic thermal sensitive uracilDNA-glycosylase in a lateral flow biosensor: application to the detection of Streptococcus pneumoniae. Mikrochimica acta. 2018; 185:212.

20. Wang H, Ma Z, Qin J, Shen Z, Liu Q, Chen X, et al. A versatile loop-mediated isothermal amplification microchip platform for Streptococcus pneumoniae and Mycoplasma pneumoniae testing at the point of care. Biosensors \& bioelectronics. 2019; 126:373-80.

\section{Tables}


Table 1. Bacteria strains used in the S. pneumoniae MCDA assay.

\begin{tabular}{|c|c|c|c|}
\hline ID & Strains & Sources & MCDA result \\
\hline 1 & Streptococcus pneumoniae & ATCC49619 & Positive \\
\hline $2-15$ & Streptococcus pneumoniae & Isolated strains from sputum & Positive \\
\hline 16 & Shigatoxin-producing E. coli (STEC) & E. Coli 933 & Negative \\
\hline 17 & Enteroaggregative E. coli (EAEC) & E. Coli 042 & Negative \\
\hline 18 & Enteroinvasive Escherichia coli (EIEC) & E. Coli 44825 & Negative \\
\hline 19 & Enteropathogen Escherichia coli (EPEC) & E. Coli $2348 / 69$ & Negative \\
\hline 20 & Enterotoxigenic Escherichia coli (ETEC) & E. Coli 10407 & Negative \\
\hline 21 & Salmonella typhi & Isolated strains from feces & Negative \\
\hline 22 & Citrobacter freundii & Isolated strains from sputum & Negative \\
\hline 23 & Listeria ivanovii & BAA678 & Negative \\
\hline 24 & Listeria monocytogenes & Isolated strains from blood & Negative \\
\hline 25 & Klebsiella pneumoniae & ATCC2146 & Negative \\
\hline 26 & Streptococcus suis & Isolated strains from feces & Negative \\
\hline 27 & Proteus mirabilis & Isolated strains from urine & Negative \\
\hline 28 & Streptococcus salivarius & Isolated strains from dialysate & Negative \\
\hline 29 & Neisseria perflava & Isolated strains (unknown) & Negative \\
\hline 30 & Staphylococcus cohnii & Isolated strains from environment & Negative \\
\hline 31 & Pseudomonas aeruginosa & Isolated strains from sputum & Negative \\
\hline 32 & Enterobacter cloacae & ATCC700323 & Negative \\
\hline 33 & Serratia marcescens & Isolated strains from sputum & Negative \\
\hline 34 & Candida krusei & Isolated strains from urine & Negative \\
\hline 35 & Bacillus cereus & Isolated strains from blood & Negative \\
\hline 36 & Escherichia coli & ATCC25922 & Negative \\
\hline 37 & Acinetobacter baumannii & Isolated strains from sputum & Negative \\
\hline 38 & Pseudomonas aeruginosa & ATCC27853 & Negative \\
\hline 39 & Escherichia coli & ATCC35218 & Negative \\
\hline 40 & Streptococcus agalactiae & Isolated strains from sputum & Negative \\
\hline
\end{tabular}


Table 2. Clinical sensitivity of $S$. pneumoniae MCDA assay in sputum samples.

\begin{tabular}{lll} 
Method & LoD & Sputum \\
\hline MCDA & $2.9 \mathrm{CFU} /$ reaction & $447 \mathrm{CFU} / \mathrm{ml}$ \\
\hline PCR & $290 \mathrm{CFU} /$ reaction & $44700 \mathrm{CFU} / \mathrm{ml}$
\end{tabular}

Table 3. Primers used in this study.

\begin{tabular}{lll} 
Primera & Sequence and modification $\left(5^{\prime}\right.$-3' $^{\prime}$ & Lengthb \\
\hline F1 & AGGATTTAAAACAGAGAGGAATT & $23 \mathrm{nt}$ \\
\hline F2 & CGCCCCCTAAAATAACCG & $18 \mathrm{nt}$ \\
\hline CP1 & GAGATAGACTTGGCGCCCATTCTGCAGAGCGTCCTTTG & $38 \mathrm{mer}$ \\
\hline C1 & GAGATAGACTTGGCGCCCAT & $20 \mathrm{nt}$ \\
\hline C1* & 5'-FITC-GAGATAGACTTGGCGCCCAT-3' & $20 \mathrm{nt}$ \\
\hline CP2 & AAGGAGTCAAGGTAGCTCCTCCCTTCACTTCTGTATTGTCCAA & $43 \mathrm{mer}$ \\
\hline C2 & AAGGAGTCAAGGTAGCTCCTC & $21 \mathrm{nt}$ \\
\hline D1 & GCAACACTCGAAATATAGAC & $20 \mathrm{nt}$ \\
\hline D1* & 5'-Biotin-GCAACACTCGAAATATAGAC-3' & $20 \mathrm{nt}$ \\
\hline D2 & AGACAGAGTGGAAGCAGA & $18 \mathrm{nt}$ \\
\hline R1 & ATCACTCTTACTCGTGGT & $18 \mathrm{nt}$ \\
\hline R2 & AGTAGAGGCTGCTTTTGAAG & $20 \mathrm{nt}$
\end{tabular}

a C1*, 5'-labeled with FITC for the MCDA-LFB assay; D1*, 5'-labeled with Biotin for the MCDA-LFB assay.

b nt, nucleotide; mer, monomeric.

\section{Figures}


A

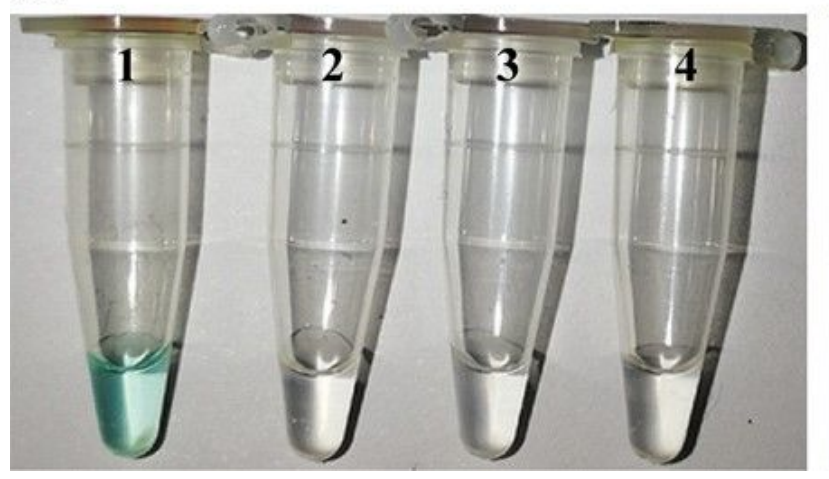

B

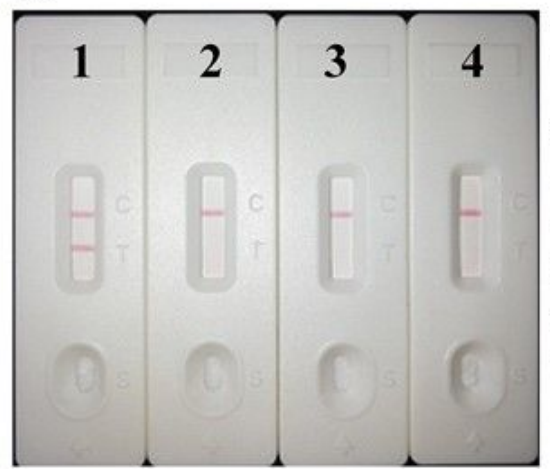

C

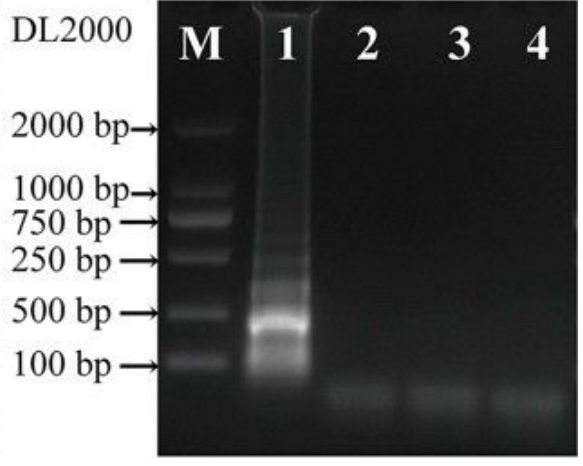

\section{Figure 1}

Confirmation of S. pneumoniae MCDA products.

A

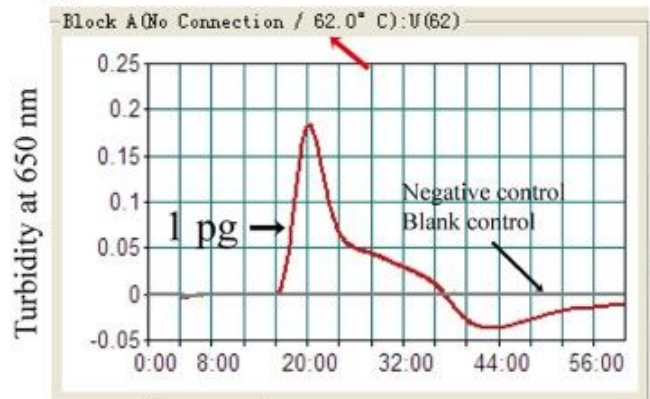

Time (minute:second)

D

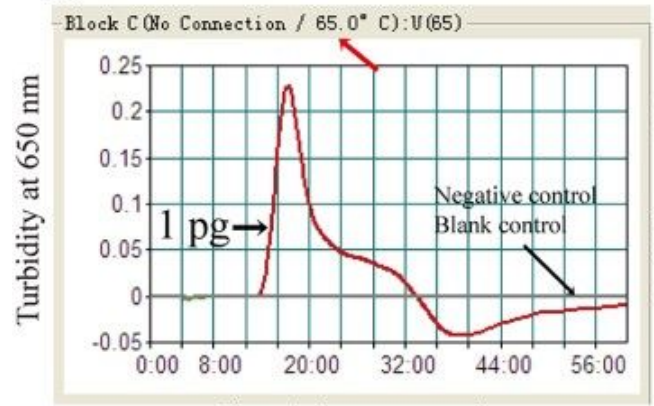

Time (minute:second)
B

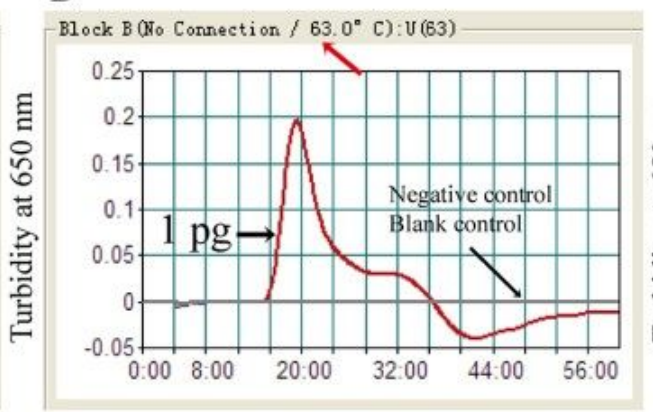

Time (minute:second)

E

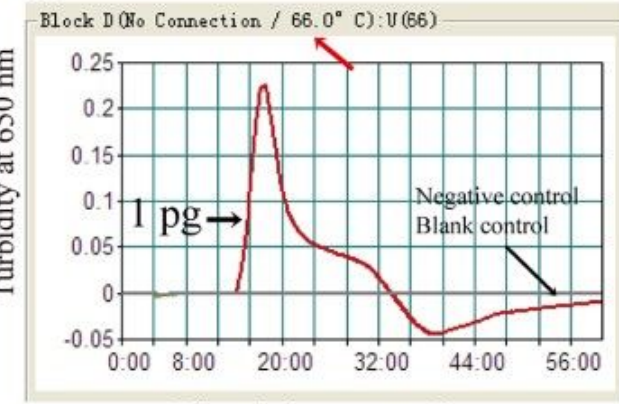

Time (minute:second)
C

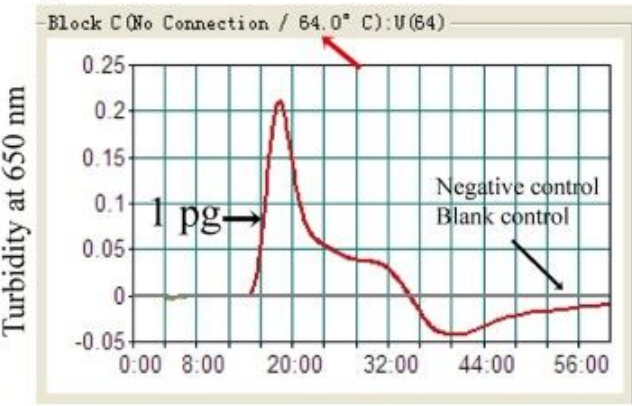

Time (minute:second)

\section{F}

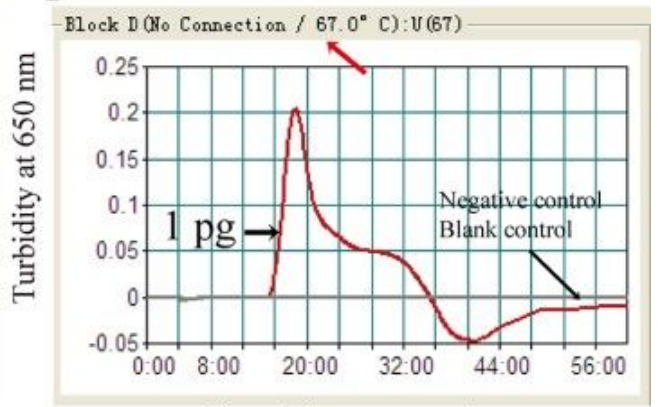

Time (minute:second)

Figure 2

Optimal temperature of S. pneumoniae MCDA assay. 
A

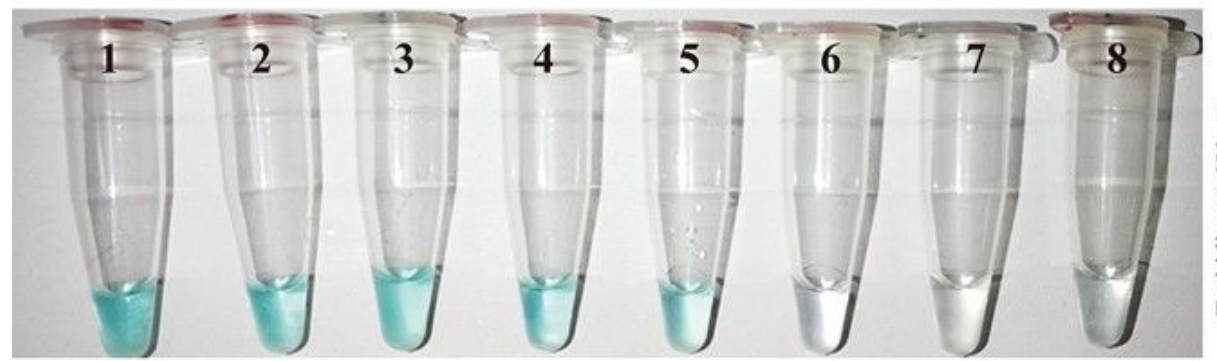

C

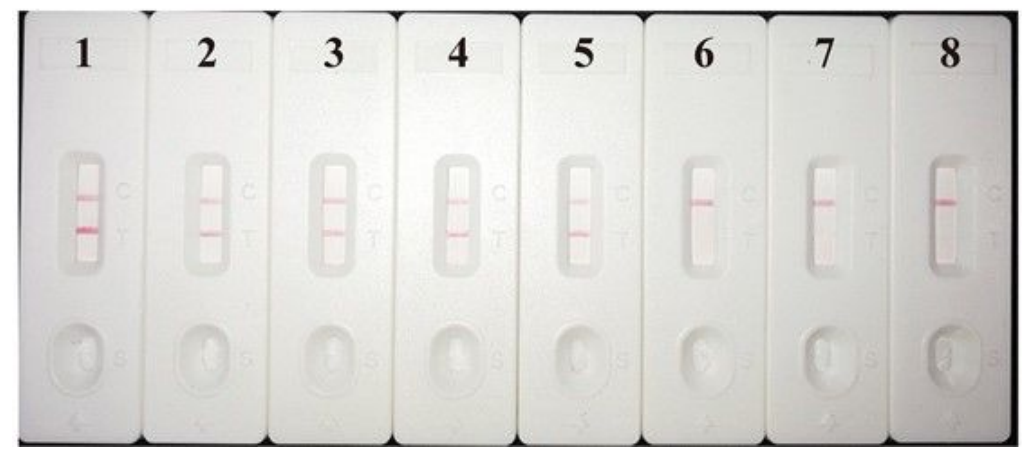

B

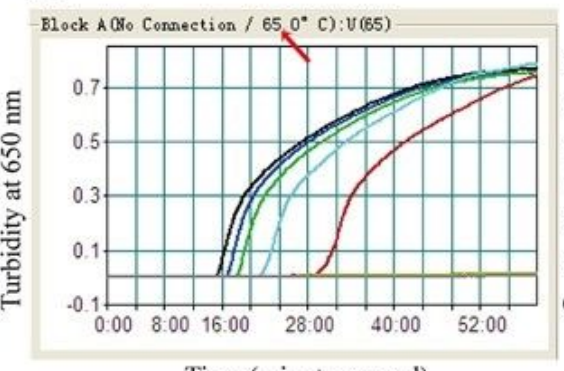

Legend -

\section{D}

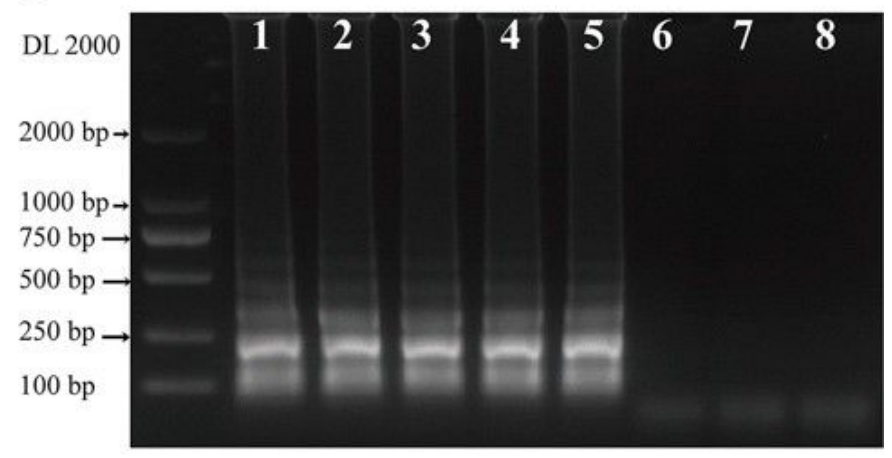

\section{Figure 3}

Sensitivity of S. pneumoniae MCDA assay. 

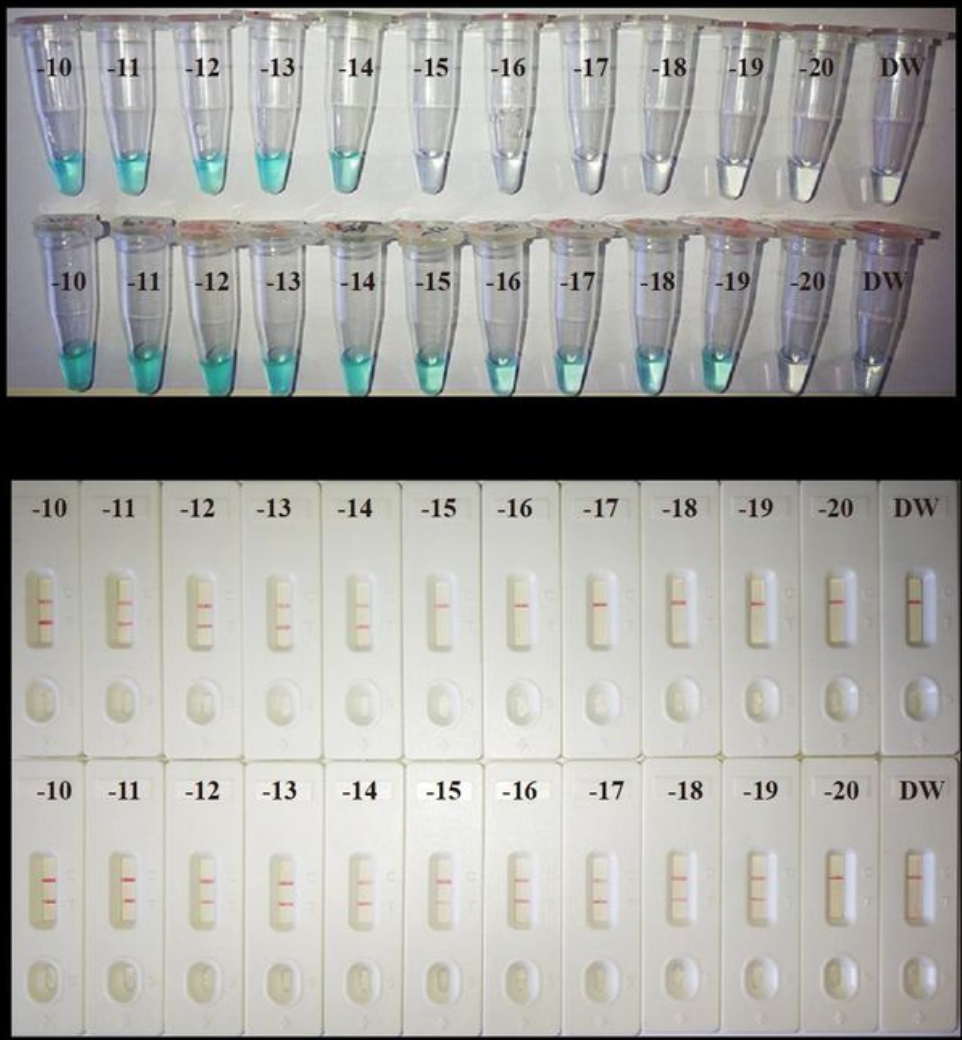

\section{Figure 4}

MCDA assay coupled with AUDG enzyme eliminates false-positive results caused by carryover contamination.

GGAGATGTGTTTCAAGATACTGTAACGGTAGAGGATTTAAAACAGAGAGGAATTTCTGCAGAGCGTCCTTTG $\stackrel{\text { D1 }}{\text { GTCTATATTTCGAGTGTTGCTTATGGGCGCCAAGTCTATCTCAAGTTGGAAACCACGAGTAAGAGTGATGA }}$ $\underset{\text { AGTAGAGGCTGCTTTTGAAGCTTTGATAAAAGGAGTCAAGGTAGCTCCTCAGACAGAGTGGAAGCAGATT }}{\stackrel{\text { R2 }}{\longrightarrow}}$

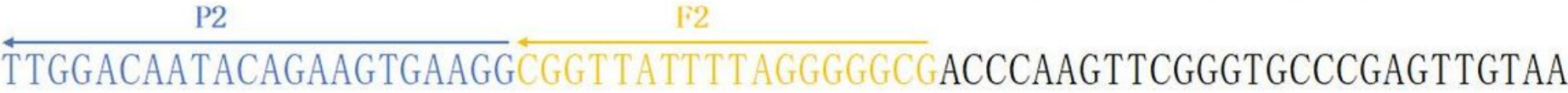

\section{Figure 5}


Location of the primers on the ply gene used for MCDA assay. 